\title{
AUTOMATED GENERATION OF MATERIAL LAWS AT LARGE STRAINS USING A GENETIC ALGORITHM
}

\author{
H. WULF ${ }^{1}$, R. KIESSLING ${ }^{1}$, R. GYPSTUHL ${ }^{1}$ AND J. IHLEMANN ${ }^{1}$ \\ ${ }^{1}$ Chemnitz University of Technology \\ Professorship of Solid Mechanics \\ Reichenhainer Str. 70, 09126 Chemnitz, Germany \\ hans.wulf@mb.tu-chemnitz.de \\ robert.kiessling@mb.tu-chemnitz.de
}

Key words: Rheological models, Material modeling, Genetic algorithm, Model identification

\begin{abstract}
This work presents an approach for automatically generating material laws at large strain. To this end, a concept of material modelling based on directly connected rheological elements is applied. This concept allows the implementation of any material law defined by a rheological model by recursive evaluation of connection relations. Therewith a tree encoding for the rheological model is applied. The search for suitable material laws fitting experimental data is realized by a genetic algorithm, which generates rheological models and evaluates their quality with respect to the given measurements. The central concepts and the genetic operators are presented in this contribution. Finally, the method is verified with synthetic data and utilized to real experimental data.
\end{abstract}

\section{INTRODUCTION}

Within the development of a material laws at large strains, rheological models are often applied to visualize the considered phenomenological effects. Afterwards, the consecutive equations are developed by formulating an ansatz for the free energy and the evaluation of the Clausius-Duhem inequality. This results in evolution equations describing inelastic effects. This approach has often been applied to formulate laws for materials having elastic, viscous and plastic properties (see, for instance, $[16,4,10,20]$ ). Contrary to this, there are approaches aiming for a direct formulation from a rheological model. To this end, kinematic as well as kinetic relations are evaluated with respect to the connected rheological elements representing nonlinear material models at large strains. By limiting the basic structure of the rheological model to a parallel connection, this method has been applied for example by [1], [9], [17] and [21]. The complexity of the rheological models and, thus, the phenomenological effects, will increase, if series connections are additionally considered. To enable the consideration of series connection, [13] derived kinetic relations for a general series connection having an approach by [12] in mind and assuming the multiplicative decomposition of the deformation gradient (see, also, [14]). Hence, every arbitrary material model at large strains based on a rheological model can be developed and implemented by evaluating the concerning connection relations.

Aiming for the simulation of a specific material behaviour, the identification of an appropriate rheological model and, thus, of a material model at large strains is still a challenging task. However, the described capability to implement a large number of models opens up the opportunity to search for good 
models automatically using a computer program. Here, a difficulty is that the search space is discrete, has a complex structure and is very large. In addition, a large number of potential solutions is infeasible. Algorithm that can deal with these challenges well are genetic algorithms. These are stochastic optimization algorithms based on the concept of evolution introduced by [11]. They have already been applied to a wide variety of engineering applications [3]. In the context of material law development, genetic algorithms have been used on several occasion for the identification of material parameters (see, i.e., $[18,2,5])$. Hence gradient-based methods stay the most common choice for this task. The development of the material laws itself is closely linked to a subarea called genetic programming (see $[15,19]$ ), dealing with the task of generating a function implementation. There are few applications to constitutive modeling, which involve finding the structure of functions within the material law (see $[7,8,6]$ ).

Contrary to this, the paper at hand is not about finding an arbitrary function, but a material law in the context of rheological models, which implies all advantages, like guaranteed thermodynamic consistency. Nevertheless, it can be seen in Sec. 2.2 that the encoding for this problem is very similar to tree-structures which are typical for genetic programming and the algorithm presented in Sec. 3 can reuse many methods from genetic programming. The results in Sec. 4 demonstrate the potential of this approach but also highlight the need for further research.

\section{MATERIAL MODELING AT LARGE STRAINS BASED ON DIRECTLY CONNECTED RHEOLOGICAL ELEMENTS}

\subsection{Connection relations and rheological elements at large strains}

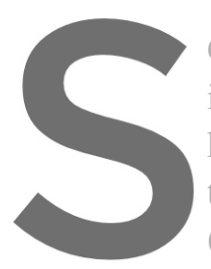

Generally, rheological in series and parallel

kinematic and kinetic rela

these connection relation

(see [13]). Concerning a sin
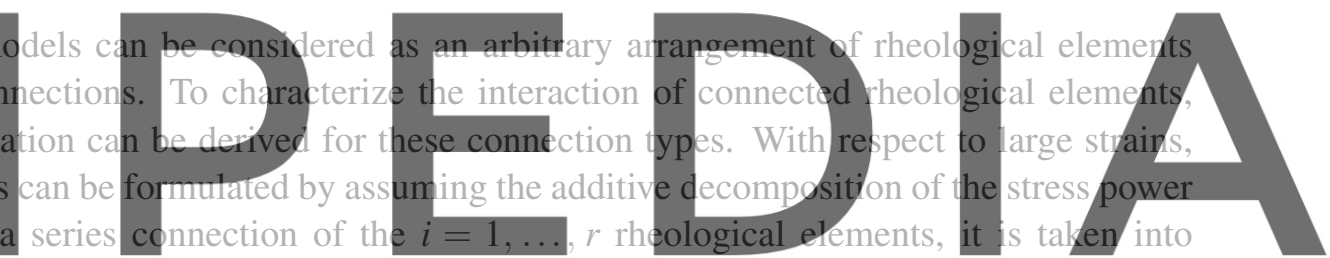

account that the associated total deformation gradient $F$ is multiplicatively decomposed into the partial

Register forf \&neeialtsh hit.

Here, $\underset{\tau}{\tau}$ and $\underset{\underline{T}}{\underline{i}}$ denote the Kirchhoff stress tensor and the second Piola-Kirchhoff stress tensor

$$
\stackrel{i}{\underline{T}}=\underline{i}^{-1} \cdot \stackrel{i}{=} \cdot\left(\stackrel{i}{F}^{T}\right)^{-1}
$$

of the $i$-th partial deformation, respectively. The contained right Cauchy-Green tensor is given by

$$
\stackrel{i}{\underline{\underline{C}}}=\underline{\underline{i}}^{T} \cdot \stackrel{i}{\underline{F}} .
$$

Furthermore, $\stackrel{R-1}{\underline{F}}$ represents a cumulated deformation gradient according to

$$
\stackrel{R-1}{\underline{F}}=\stackrel{r-1}{\underline{F}} \cdot \cdots \cdot \stackrel{2}{\underline{F}} \cdot \stackrel{\underline{F}}{\underline{F}} .
$$

Note, that the kinetic relations, given in Fig. 2, represent stress equilibria on the intermediate configurations as well as on the current configuration of the related cumulated deformation. 


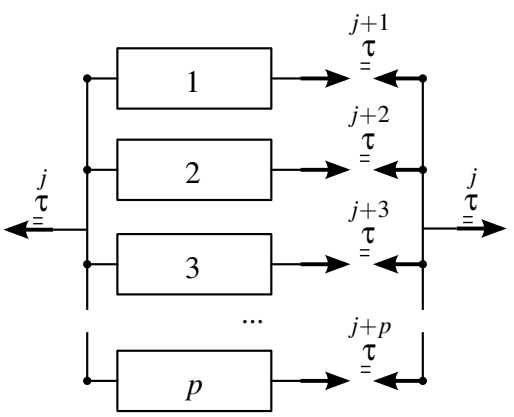

Kinematic relation:

$\stackrel{j}{\underline{F}}=\stackrel{j+1}{=} \stackrel{j}{=} \stackrel{j+2}{\underline{F}}=\ldots=\stackrel{j+p}{F}$

Kinetic relation:

$\underset{\tau}{=}=\sum_{i=j+1}^{j+p} \stackrel{i}{\tau}$

Figure 1: Kinematic and kinetic relations of a parallel connection of $j$ rheological elementes
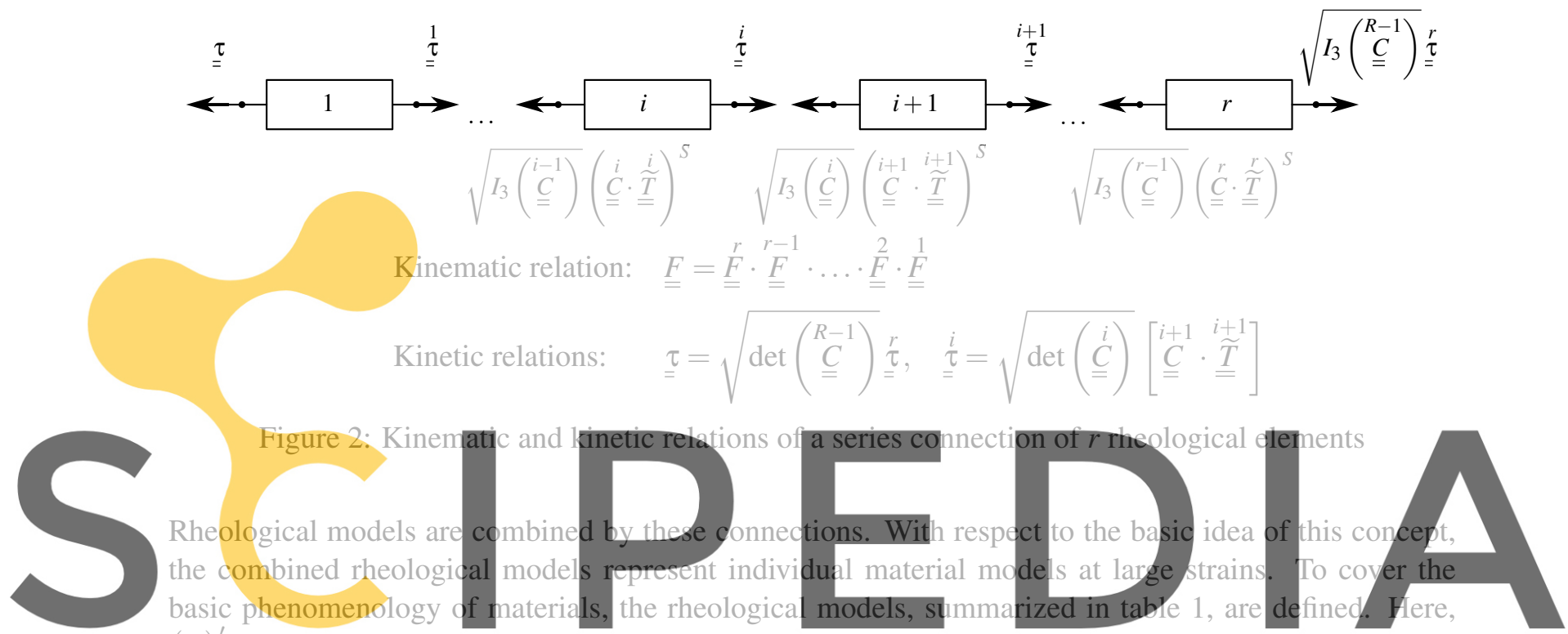

$(\underline{X})^{\prime}$ represents the deviatoric part of a second-rank tensor $\underline{X}$. The Lagrangian material derivative of $\underline{C}$

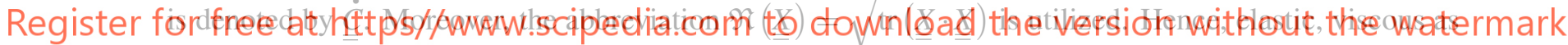
well as plastic effects can be considered. Furthermore, the endochronic element is introduced to simulate the effect of kinematic hardening. Note, that the quantities indicated by an asterisk represent parameter of the rheological model.

\subsection{Tree encoding of rheological models}

The presented method allows the implementation of a arbitrary material model that takes any rheological model as an input and evaluates this model for a given loading schedule. This requires a data structure to represent the rheological model in order to pass it as an input variable. Due to the recursive and hierarchical nature of rheological models, a tree structure is the natural choice. Therein, the inner nodes represent the parallel and serial connections. The subtrees of each inner node are the components of the connection. These subtrees may be leaf nodes which represent the basic elements or branched trees themselves resulting in a nested model. In Fig. 3 a simple model and its corresponding tree representation is shown as an example. In Tab. 2 the integer encoding of the node types is listed. Obviously, the inner nodes are always strictly negative, whereas the leaf nodes have positive numbers. The tree representation can be transformed into an integer list by prefix serialization, which is always listing the root node first 
Table 1: Rheological elements representing individual material laws at large strains

\begin{tabular}{|c|c|}
\hline isotropic hyperelasticity & $\begin{array}{l}\underline{\underline{\widetilde{T}}}=2 \frac{\partial \widetilde{\rho} \psi\left(J_{1}, J_{2}, J_{3}\right)}{\partial \underline{\underline{C}}}, \text { with } J_{3}=\sqrt{\operatorname{det}(\underline{\underline{C}})} \\
\underline{\underline{\bar{C}}}=J_{3}^{-2 / 3} \underline{\underline{C}}, J_{1}=\operatorname{tr}(\underline{\underline{\underline{C}}}), J_{2}=\frac{1}{2}\left[J_{1}^{2}-\operatorname{tr}\left(\underline{\underline{\underline{C}}}^{2}\right)\right]\end{array}$ \\
\hline viscous flow & $\underline{\underline{\underline{T}}} \cdot \underline{\underline{C}}=\eta^{*}\left(\frac{1}{k_{0}} \mathfrak{N}(\underline{\underline{\widetilde{T}}} \cdot \underline{\underline{C}})\right)^{N^{*}-1}\left(\underline{\underline{C}}^{-1} \cdot \underline{\underline{\dot{C}}}\right)^{\prime}, k_{0}=1 \mathrm{MPa}$ \\
\hline$\stackrel{\text { plastic flow }}{\searrow}\ulcorner$ & $\begin{array}{l}\underline{\underline{C}}^{-1} \cdot \underline{\underline{\dot{C}}}=\chi \underline{\underline{\widetilde{T}}} \cdot \underline{\underline{C}}, \chi \Phi=0, \chi \geq 0, \Phi \leq 0 \\
\text { with, e.g., } \quad \Phi=\mathfrak{N}\left((\underline{\underline{T}} \cdot \underline{\underline{C}})^{\prime}\right)-\sqrt{\frac{2}{3}}\left[\sigma_{F}^{*}+R(\underline{\underline{C}})\right]\end{array}$ \\
\hline endochronic element & $\begin{array}{l}\underline{\underline{\widetilde{T}}} \cdot \underline{\underline{C}}=\mu^{*}\left(\underline{\underline{C}}_{1}^{-1} \cdot \underline{\underline{\bar{C}}}\right)^{\prime}, \quad \underline{\underline{\underline{C}}}_{1}=\frac{\mu^{*} k_{t} \dot{\varphi}}{\eta^{*}} \underline{\underline{C}}_{1} \cdot\left(\underline{\underline{C}}_{1}^{-1} \cdot \underline{\underline{\bar{C}}}\right)^{\prime} \\
\text { with } \quad \dot{\varphi}=\sqrt{\frac{1}{6}} \mathfrak{N}\left(\underline{\underline{C}}^{-1} \cdot \underline{\underline{\dot{C}}}\right), k_{t}=1 \mathrm{~s}\end{array}$ \\
\hline
\end{tabular}

and the listing the subtrees recursively in the same manner. Before listing the subtrees, an integer is inserted denoting the number of subtrees. The result for the example tree given in Fig. 3 is $[-220-$ 1210 . Overall, this gives an integer list format, which can be easily passed as a function parameter.
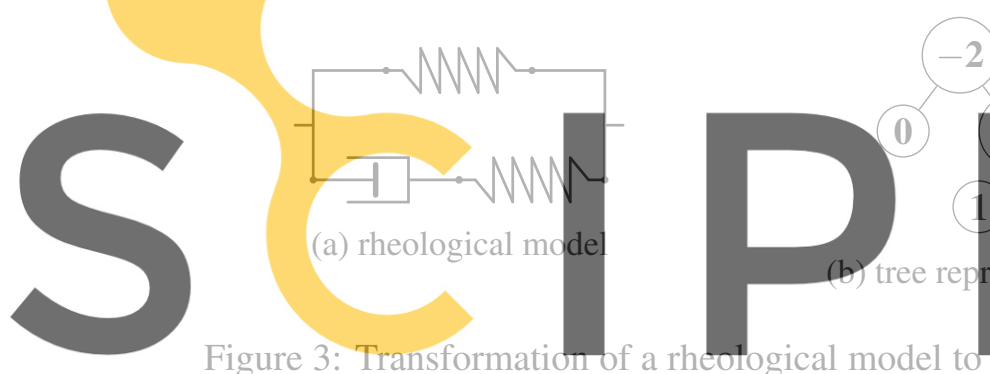

The integer list representation is $-220-12101$

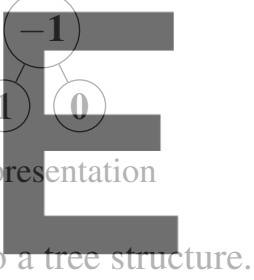

Register for free at https//www.scipedia.com to
Table 2: Integers encoding for the node types

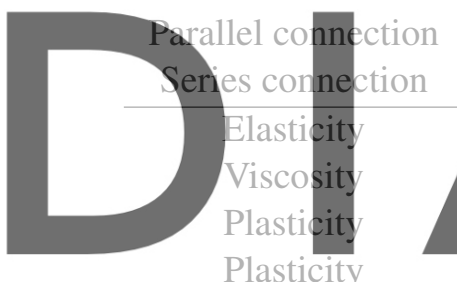
download the version waxwell model 3 itheyt the watermark

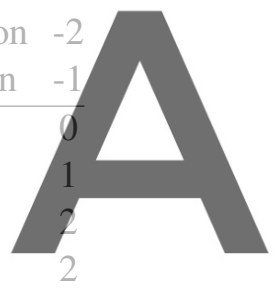
download the version withoyt the watermark

\subsection{Black Box for material model evaluation and parameter identification}

The usual objective is to fit the material model to a set of experimental data, which is achieved by an optimization algorithm. To this end, a program extracts the loading schedule from the experimental data and computes the stress-strain relation for a given material model. The result is compared to the measurements via standard least-squares fit and the material parameters are optimized by the LevenbergMarquardt algorithm. Overall, the user has to supply the encoded rheological model, the initial model parameters and the experimental data. The identification program will return the optimized model parameters and a squared error sum indicating the fitting quality of the model.

No knowledge about the internals of the program and little knowledge about material modeling is required to utilize this. Instead, it can be treated as a "Black box" that takes rheological models as input and returns an assessment of the quality of the model for describing the measured material. Therefore, it is very easy to test and evaluate a large number of material models for a given set of experimental data. 


\section{GENERATION OF MATERIAL MODELS WITH A GENETIC ALGORITHM}

\subsection{General concept}

The methods presented in the previous section allow for testing a large number material models. The disadvantages of large-scale manual testing include the usually cumbersome data management, the risk to miss unexpected combinations and the need for manual work itself. Hence, an automated search method has to be preferred. An exhaustive search (brute force testing of all possible models up to a certain size limit) is only feasible for a very small model size, because the number of models grows exponentially with the size limit. Hence, for complex models, a more targeted search method is required.

A genetic algorithm is a stochastic optimization method that is well suited for searching a discrete and structured search space as given here. Its basic assumption is that improved solutions can be constructed by slightly modifying or recombining building blocks from already existing good solutions. The strategy for manually constructing models often involves recombining and modifying building blocks from experience or existing models. Therefore this assumption seems very well justified.

The genetic algorithm is inspired by the process of evolution and its remarkable capability concerning the adaption of species to their environment. Hence, the single solutions are called individuals and the whole set of solutions is called population in this context. The initialization of the algorithm consists of generating an initial population at random. Here, an upper limit for the size of the material models is prescribed as well as a probability distribution for the different basic elements. For each individual, a fitness value is computed, which expresses the solution quality of the associated material model. In

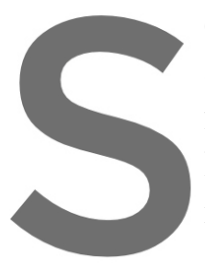
order to evaluate the model quality, the genetic algorithm calls the
as a subroutine to compute a parameter fitting and a squared error
from the squared error sum (whose minimization is the primary
like model size and complexity. New individuals are created by
multiple parent individuals and create one or multiple offspring selected from the current population

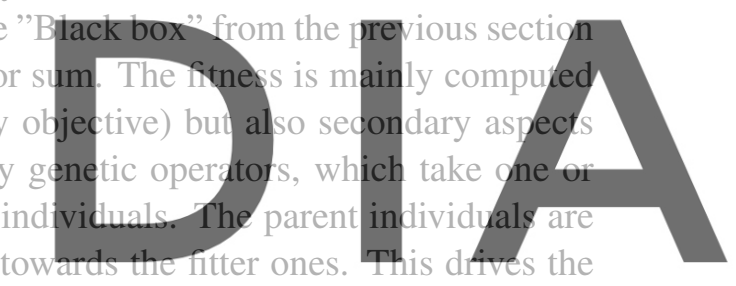

new population with the same size as the previous is completed and replaces the previous population. However, the applied algorithm only generates individuals up to a fraction of the overall population size (usually around 25\%) and replace the least fit individuals. This offers the individuals with a high fitness a significantly increased survival time, providing a second incentive for improvement. This method yields a way more targeted search than the standard approach and is capable for finding good solutions with a moderate number of model evaluations in the magnitude of 100 to 500. The algorithm terminates when the maximum number model evaluations is reached or when a model with a squared error sum below a given threshold is found. The result is the best model found so far, but additionally examining the second and third best is often worthwhile.

\subsection{Genetic operators}

\section{Selection}

The standard roulette wheel selection is used as the selection method (see [11]). That means, that the probability $p_{i}$ to be selected for each individual $i$ is computed from its fitness $f_{i}$ by 


$$
p_{i}=\frac{f_{i}}{\sum_{i} f_{i}} \quad \text { with } \quad f_{i}=\frac{1}{r_{i}+p_{i}} .
$$

The fitness is computed from the squared error sum $r_{i}$ and some penalty terms $p_{i}$. Those penalty terms are set as follows.

- A very high penalty for models that fail the parameter identification is selected.

- A penalty proportional to the number of basic elements is determined. Hence, simple models are preferred.

- A penalty for models exceeding a limit in depth of nested connections is defined. Complexity in the form of deeply nested connections is usually especially undesirable.

- The number of very similar or identical individuals in the population is penalized. Having multiple copies of the same models reduces the genetic variability and is therefore detrimental.

The penalty term must be scaled to fit the magnitude of $r_{i}$. Moreover, the selection probability is capped to prevent domination of a single individual.

\section{Mutation}

The mutation operator takes one individual as input and modifies the rheological model slightly to generate a new one. Its main objective is to explore the search space. As an example, the add-to-leaf mutation
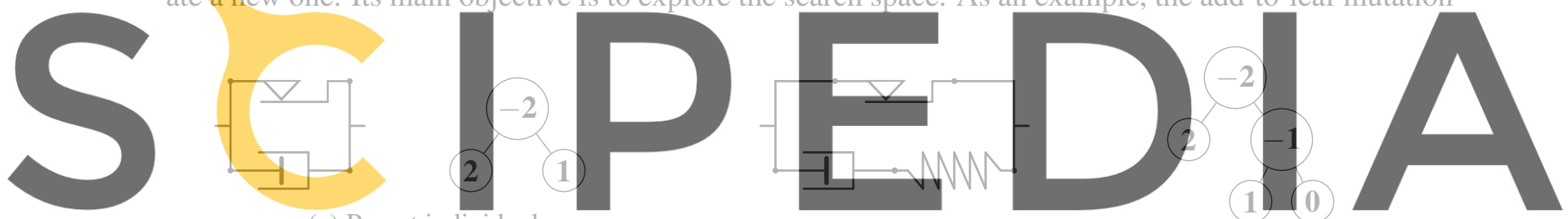

(a) Parent individual

(b) Offspring individual

Register for free at https//www.scipedia.com to download the version without the watermark

Figure 4: add-to-leaf mutation operator: A leaf node is expanded into a connection and a random addi-

tional leaf node is added to this connection.

operator is shown in Fig. 4. It chooses one leaf node at random and inserts a new connection node at its place. The type of the connection is always opposite to the connection node above or random at the root. The leaf node is appended to this connection node together with a newly generated basic element with random type. The probability distribution for the element type is uniform at the start, but can be adaptively changed during the run. A good idea is to shift the probability distribution towards the actual distribution within the population. Due to this, fewer of those will be newly generated, if the certain element type is filtered out because it is detrimental for a given problem. In general, adaptive operators can considerably improve the performance of the algorithm.

Other mutation operators, being utilized, are the following.

- Add a new leaf to a connection.

- Remove a leaf node. 
- Swap two nodes or subtrees.

- Change the type of a leaf node. Alternatively, the material model can be changed, for instance the main type is kept as elasticity, but the ansatz for the free energy $\widetilde{\rho} \psi$ function is modified. Exemplary, a change from the Neo-Hooke ansatz to the Mooney-Rivlin ansatz is possible.

\section{Crossover}

The crossover operator takes two individuals as input and generates two new ones. Its main objective is to recombine the building blocks of successful individuals and thereby exploit the knowledge about good building blocks, that has been accumulated in the population. Here, the subtree crossover operator is used. It works be choosing a cutting point in the tree representation of both parent models at random. Thereafter, the subtrees below the cutting point are exchanged and two new individuals arise (see Fig. $5)$.
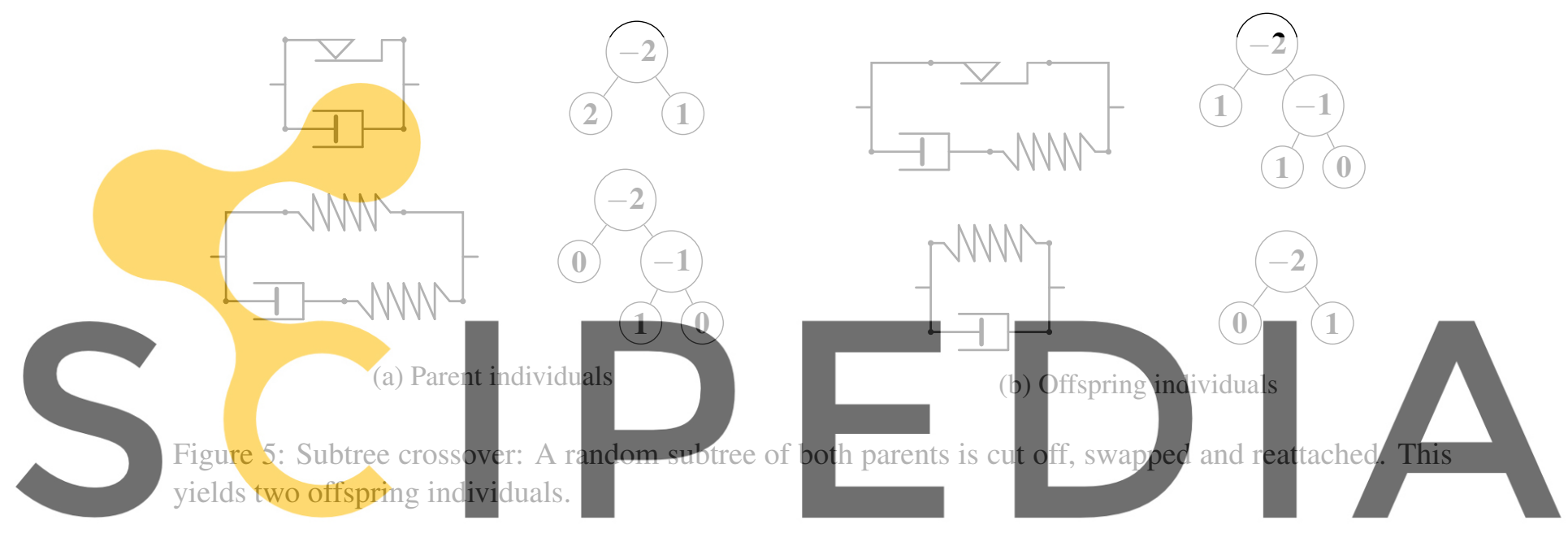

Register for free at https//www.scipedia.com to download the version without the watermark 3.3 Repairing malformed models

The application of the operators frequently yields to tree structures, which represent no valid model or contain unnecessary complexity. Therefore, checking all new models and repairing, if required, is essential. To repair a model, the following operations can be applied.

- Connections with only one sub-node are removed, then their subtree is shifted up one layer.

- Directly nested connections of the same type are merged together. As a result, the type of the connections always flips between adjacent layers.

- The basic elements within each connection are ordered.

- Directly adjacent basic elements of the same type within the same connection are merged.

In general, basic elements can be merged only if the material laws fully coincide. Occasionally, merging different models is possible, for instance an elastic element based on the Neo-Hookean ansatz parallel to an elastic element based on the Mooney-Rivlin ansatz can be merged into a single elastic element based on the Mooney-Rivlin ansatz. 


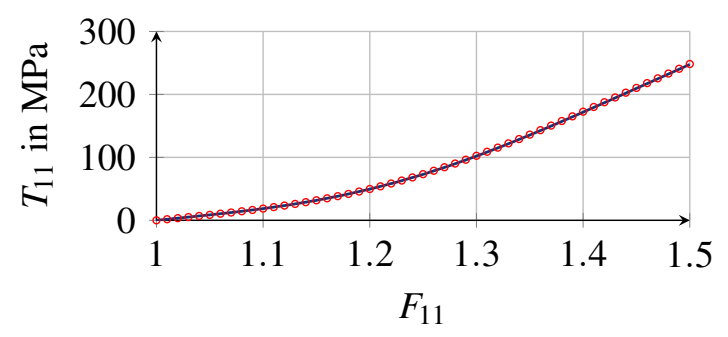

(a) Tension test

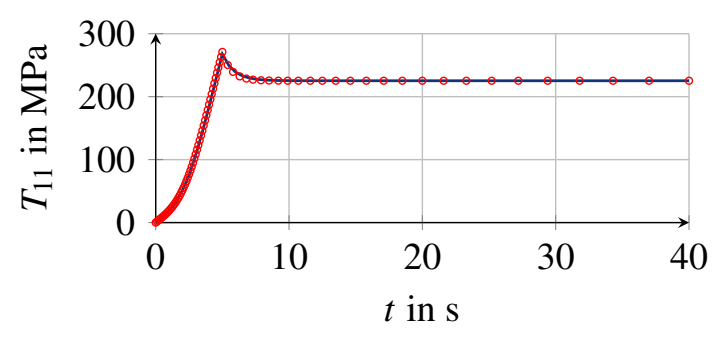

(b) Relaxation test

Figure 6: Synthetic data generated from the Zener model. In addition, the best model fit is shown. As the original model was recovered, the fit is perfect except for noise.

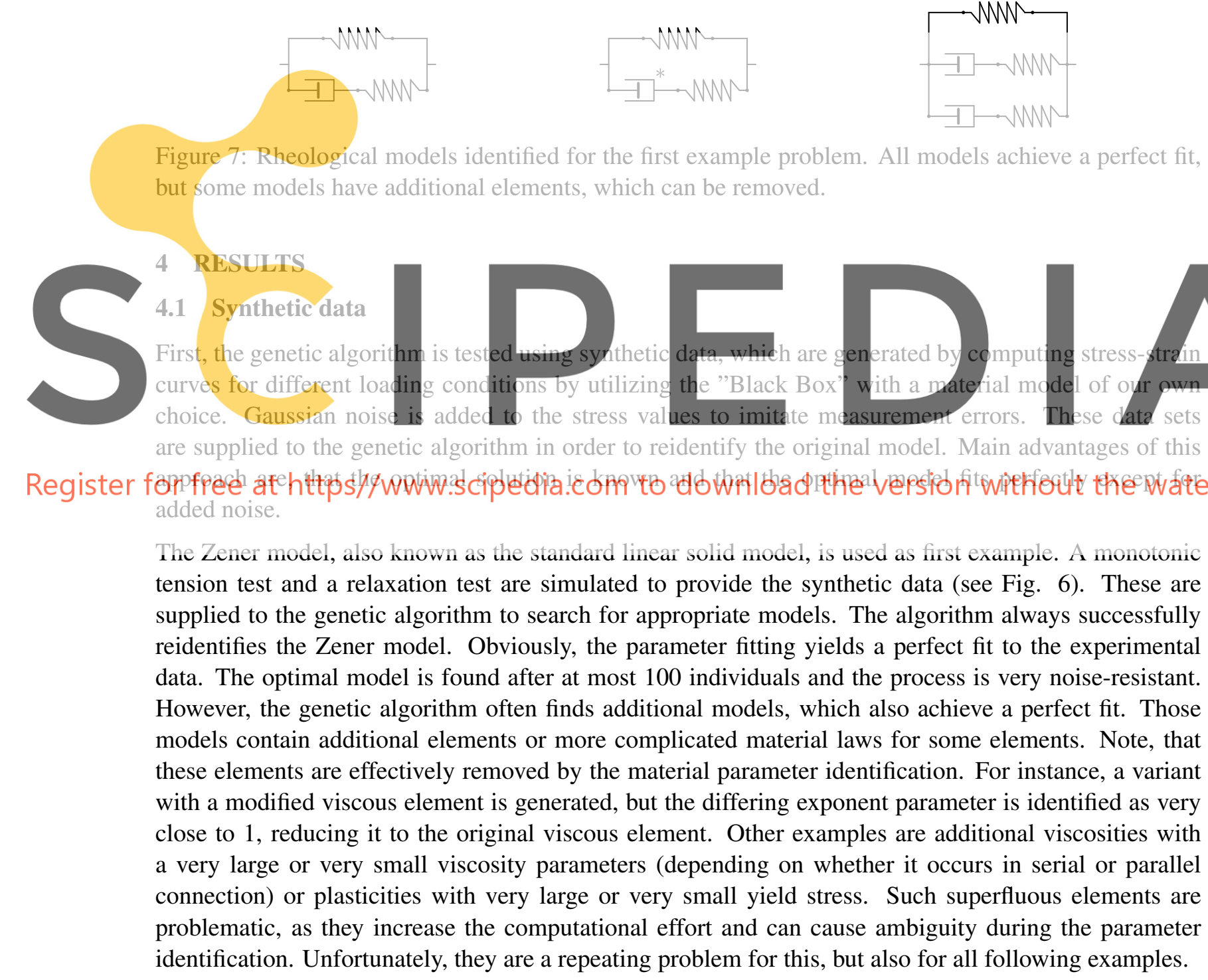




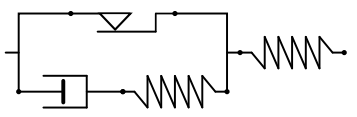

Figure 8: The Schwedoff model, which was used to generate the synthetic data for the second example problem. The genetic algorithm managed to reidentify this model using the small strain implementation.

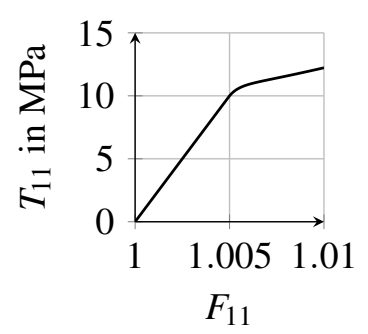

(a) Tension test at strain rate $5 \cdot 10^{-2} \mathrm{~s}^{-1}$

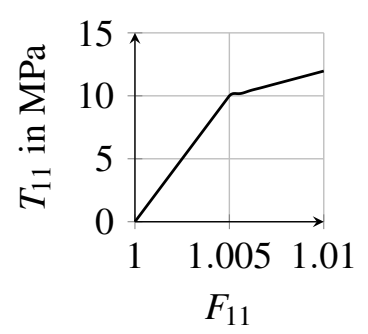

(b) Tension test at strain rate $5 \cdot 10^{-4} \mathrm{~s}^{-1}$

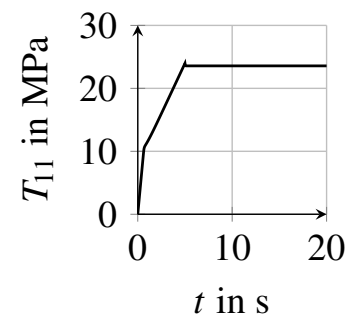

(c) Relaxation test

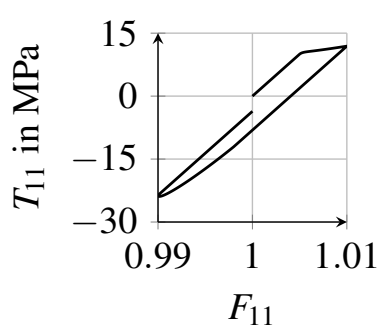

(d) Cyclic test

Figure 9: Synthetic data generated from the Schwedoff model. As the exact model is reidentified, the best fit exactly matches these curves and is therefore omitted.

The second example is a Schwedoff model. Here, monotonic tests at two different strain rates $\left(5 \cdot 10^{-2} \mathrm{~s}^{-1}\right.$ and $5 \cdot 10^{-4} \mathrm{~s}^{-1}$ ), a relax

existing small strain impl algorithm converges even ments are useful here. A

Applying the large strain parameter identification procedure, which fails or

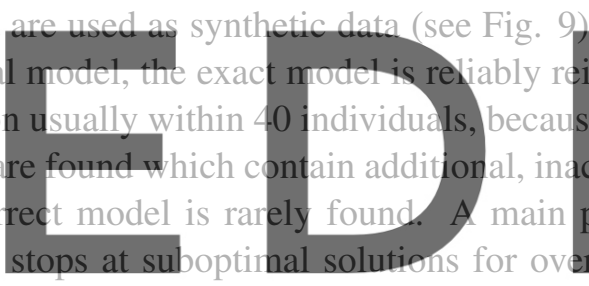
Utilizing the
se all basic ele-
ctive elenents.
problem is the
er-paranetrized models. Moreover, the parameter identification is very sensitive to the initial parameter set. In conse-

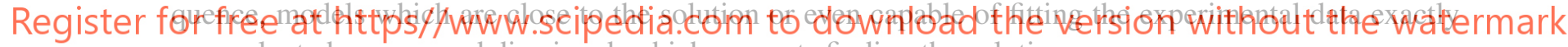
are evaluated as poor and dismissed, which prevents finding the solution.

\subsection{Experimental data}

The method is also applied to experimental data achieved from the investigation of the polymer BASF Ultramid B40. The first dataset consists of a monotonic and a relaxation test as shown in Fig 10. The genetic algorithm identifies an extended Maxwell model with two Maxwell models, which are series connections of visous and elestic elements, as the best solution. A typical variant that is found uses an elastic element with the Mooney-Rivlin energy approach instead of the Neo-Hookean approach for one of the Maxwell models. The results from the evaluation of the fitted material laws are also shown in Fig 10. It has to be mentioned, that the extended Maxwell model would also have been the manual choice based on the experimental data. Hence, finding it with the genetic algorithm is very reassuring and this example can be considered as a success.

As a final example, the full set of data from the experimental investigation of the polymer are considered. This comprises monotonic tests at three different strain rates $\left(1 \cdot 10^{2} \mathrm{~s}^{-1}, 5 \cdot 10^{-2} \mathrm{~s}^{-1}\right.$ and $\left.1 \cdot 10^{-4} \mathrm{~s}^{-1}\right)$ and a relaxation test (Fig. 12). At this point, the material model shown in Fig. 13 is identified using the small 


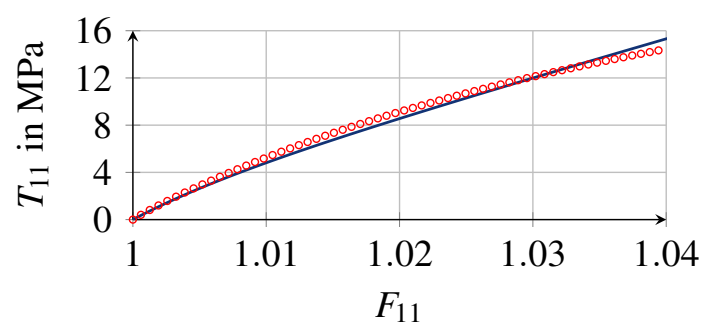

(a) tension test

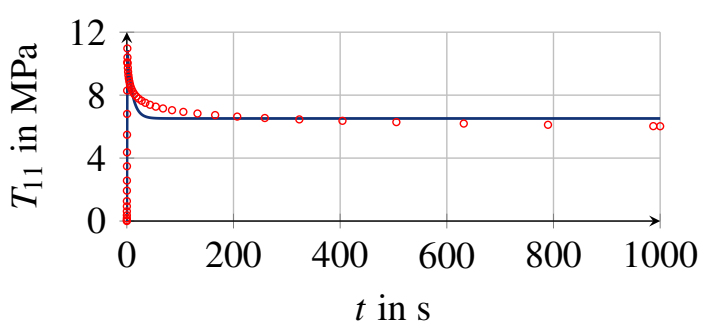

(b) relaxation test

Figure 10: Reduced viscoelastic set of experimental data (red) and the best model fit (blue)
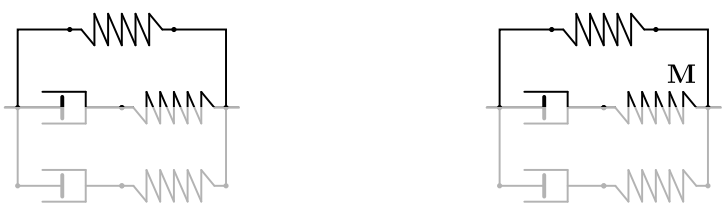

Figure 11: Extended Maxwell models are identified from the reduced viscoelastic set of experimental data. A typical variation contains the Mooney-Rivlin ansatz for the free energy of the elastic element as denoted by the superscript M.

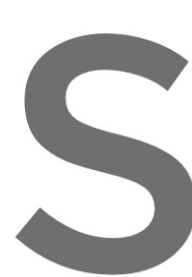

strain implementation.

used to fit some effects,

strain variant did not yic

must be considered as un
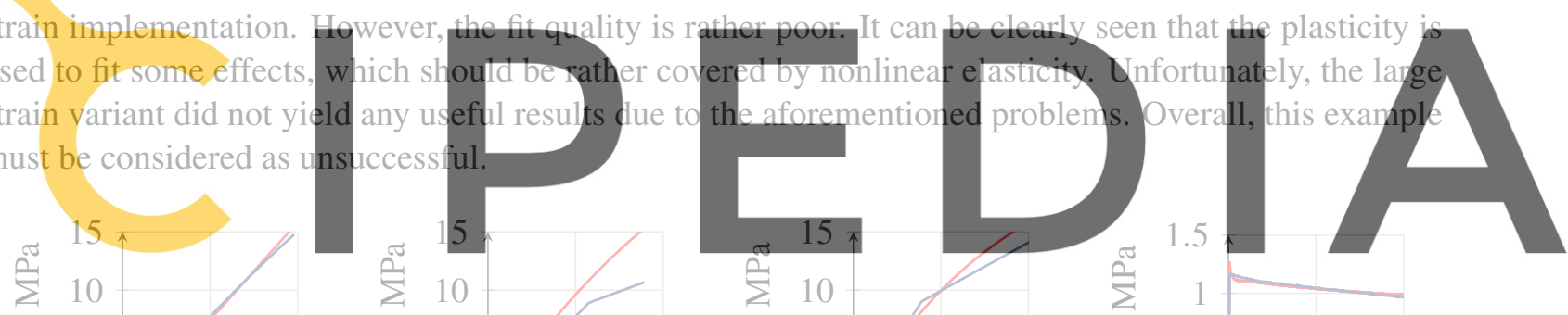

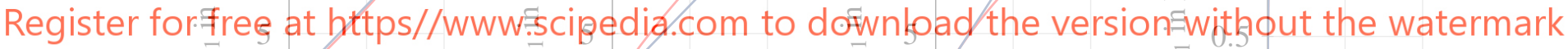

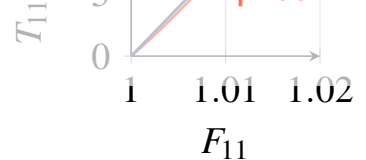

$F_{11}$
0

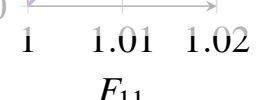

(a) Tension test at strain rate $1 \cdot 10^{2} \mathrm{~s}^{-1}$

(b) Tension test at strain rate $5 \cdot 10^{-2} \mathrm{~s}^{-1}$
(

$1 \quad 1.02 \quad 1.03$

$F_{11}$
$0 \quad 15003000$

$t$ in $\mathrm{s}$
(c) Tension test at strain
(d) Relaxation test

Figure 12: Full set of experimental data resulting from the investigation of BASF Ultramid B40 (red) and the best model fit (blue)

\section{CONCLUSIONS and OUTLOOK}

The two main components presented in this work are the concept of material modeling, which allows for an implementation of any material model given by a rheological model, and a genetic algorithm, which allows an automated search for a good model with respect to a given set of experimental data. The 


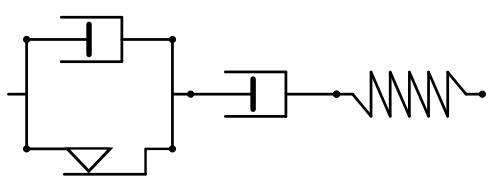

Figure 13: Model identified from the full set of the experimental data from the investigation of BASF Ultramid B40.

combination of these parts have the potential to significantly change the process of material modeling. Instead of manually designing material models, good models can automatically be generated, which reduces both the time and the knowledge required by the user. Moreover, this approach has the chance to find models that could have been missed by a manual search due to their unusual structure.

For the simple test cases with synthetic data, the correct model could be easily reidentified. Moreover, a realistic model was found when the algorithm was applied to measurements with rather simple (mainly viscoelastic) material behavior. These examples show that the method already works for less challenging test cases, which provides a proof of the concept. The application to large strain viscoplasticity yields results, which are not satisfying. The algorithm failed to find a model with sufficient fit quality and often did not find a usable model at all. A main problem source is that the genetic algorithm generates overparametrized models or models with a strange structure leading to various problems during the parameter identification.

Overall, the approach is promising, but requires improvement and further research. The method for the parameter identification should be modified to deal better with the aforementioned challenges and allow for a more stable fitting. In addition, the genetic algorithm offers a wide range of potential improvements. A variety of other operators could be tested, for instance a different crossover operator. Moreover, modified or adaptive genetic operators should be examined. In order to achieve a more focused search, a method for identifying and removing inactive model parts is an other required future step.

\section{REFERENCES}

[1] J. S. Bergström and M. C. Boyce. Constitutive modeling of the large strain time-dependent behavior of elastomers. Journal of the Mechanics and Physics of Solids, 46(5):931-954, 1998.

[2] B. M. Chaparro, S. Thuillier, L. F. Menezes, P.-Y. Manach, and J. V. Fernandes. Material parameters identification: Gradient-based, genetic and hybrid optimization algorithms. Computational Materials Science, 44(2):339-346, 2008.

[3] D. Dasgupta and Z. Michalewicz. Evolutionary algorithms in engineering applications. SpringerVerlag, Berlin and Heidelberg, 2013.

[4] W. Dettmer and S. Reese. On the theoretical and numerical modelling of Armstrong-Frederick kinematic hardening in the finite strain regime. Computer Methods in Applied Mechanics and Engineering, 193(1-2):87-116, 2004.

[5] N. Dusunceli, O. U. Colak, and C. Filiz. Determination of material parameters of a viscoplastic model by genetic algorithm. Materials \& Design, 31(3):1250-1255, 2010.

[6] X.-T. Feng and C. Yang. Coupling recognition of the structure and parameters of non-linear constitutive material models using hybrid evolutionary algorithms. International Journal for Numerical 
Methods in Engineering, 59(9):1227-1250, 2004.

[7] A. H. Gandomi and G. J. Yun. Coupled selfsim and genetic programming for non-linear material constitutive modelling. Inverse Problems in Science and Engineering, 23(7):1101-1119, 2015.

[8] A. Garg, A. Garg, K. Tai, S. Barontini, and A. Stokes. A computational intelligence-based genetic programming approach for the simulation of soil water retention curves. Transport in Porous Media, 103(3):497-513, 2014.

[9] Q. Ge, X. Luo, C. B. Iversen, H. B. Nejad, P. T. Mather, M. L. Dunn, and H. Jerry Qi. A finite deformation thermomechanical constitutive model for triple shape polymeric composites based on dual thermal transitions. International Journal of Solids and Structures, 51:2777-2790, 2014.

[10] M. E. Gurtin and L. Anand. The decomposition $\mathbf{F}=\mathbf{F}^{\mathrm{e}} \mathbf{F}^{\mathrm{p}}$, material symmetry, and plastic irrotationality for solids that are isotropic-viscoplastic or amorphous. International Journal of Plasticity, 21:1686-1719, 2005.

[11] J. H. Holland. Adaptation in Natural and Artificial Systems. MIT Press, Michigan, 1975.

[12] J. Ihlemann. Beobachterkonzepte und Darstellungsformen der nichtlinearen Kontinuumsmechanik, volume 340 von Fortschritt-Berichte VDI: Reihe 18, Mechanik, Bruchmechanik. VDI-Verlag, Düsseldorf, 2014.

[13] R. Kießling. Modellierung des mechanischen Verhaltens der Komponenten eines intrinsischen Hybridverbundes. Phd thesis, Chemnitz University of Technology, 2020.

[14] R. Kießling, R. Landgraf, R. Scherzer, and J. Ihlemann. Introducing the concept of directly connected rheological elements by reviewing rheological models at large strains. International Journal of Solids and Structures, 97:650-667, 2016.

[15] J. R. Koza and J. R. Koza. Genetic programming: on the programming of computers by means of natural selection. MIT Press, Michigan, 1992.

[16] A. Lion. Thixotropic behaviour of rubber under dynamic loading histories: Experiments and theory. Journal of the Mechanics and Physics of Solids, 46(5):895-930, 1998.

[17] M. Makki, G. Ayoub, H. Abdul-Hameed, F. Zaïri, B. Mansoor, M. Naït-Abdelaziz, and M. Ouderni. Mullins effect in polyethylene and its dependency on crystal content: A network alteration model. Journal of the Mechanical Behaviour of Biomedical Materials, 75:442-454, 2017.

[18] S. Pal, G. W. Wathugala, and S. Kundu. Calibration of a constitutive model using genetic algorithms. Computers and Geotechnics, 19(4):325-348, 1996.

[19] R. Poli, W. B. Langdon, N. F. McPhee, and J. R. Koza. A field guide to genetic programming. Lulu Enterprises, Morrisville, 2008.

[20] A. V. Shutov and R. Kreißig. Finite strain viscoplasticity with nonlinear kinematic hardening: Phenomenological modeling and time integration. Computer Methods in Applied Mechanics and Engineering, 197:2015-2029, 2008.

[21] J. Zhou, L. Jiang, and R. E. Khayat. A micro-macro constitute model for finite-deformation viscoelasticity of elastomers with nonlinear viscosity. Journal of the Mechanics and Physics of Solids, 110:137-154, 2018. 